\title{
RISK OF INFERTILITY IN PATIENTS WITH CELIAC DISEASE: a meta-analysis of observational studies
}

\author{
Juan Sebastian LASA, Ignacio ZUBIAURRE and Luis Oscar SOIFER
}

\begin{abstract}
Context - Celiac disease is an autoimmune disorder of the small intestine associated with several extra-intestinal features, such as reproductive disorders. The relationship between celiac disease and infertility has been previously assessed, with conflicting results. Objective - We seek to determine the relationship between celiac disease and infertility. Methods - Data was extracted from case-control or cohort design studies from 1966 to December 2013 using the MEDLINE-Pubmed, EMBASE, LILACS and Cochrane Library databases. We analyzed two kinds of trials: those assessing the risk of infertility in subjects with already diagnosed celiac disease, and those evaluating the prevalence of undiagnosed celiac disease in subjects with a diagnosis of infertility. Results - The search yielded 413 potentially relevant studies for revision, 12 of which were finally included for analysis. A significant association was found between women with a diagnosis of infertility and undiagnosed celiac disease [OR 3.09 (95\% CI 1.74-5.49)]. When considering those studies assessing the occurrence of infertility in subjects with already-diagnosed celiac disease, no difference was found between celiac disease patients and control subjects [OR 0.99 (0.86-1.13)]. Conclusion - Undiagnosed celiac disease is a risk factor for infertility. Women seeking medical advice for this particular condition should be screened for celiac disease. Adoption of a gluten-free diet could have a positive impact on fertility in this group of patients.
\end{abstract}

HEADINGS - Celiac disease. Infertility female. Diet gluten-free.

\section{INTRODUCTION}

Celiac disease (CD) is an autoimmune disorder triggered by intestinal exposure to gluten, a protein commonly found in wheat, barley and rye. Typical manifestation of CD consists of early onset of malabsortion-related symptoms, while atypical forms of the disease are commonly diagnosed during adulthood and frequently related to extra-intestinal manifestations $^{(8)}$.

Several extra-intestinal features have been associated with CD, such as metabolic osteopathy, anemia, and dermatitis herpetiformis ${ }^{(23)}$. There has been evidence indicating a link between $\mathrm{CD}$ and reproductive disorders, such as recurrent abortions ${ }^{(3)}$.

The relationship between $\mathrm{CD}$ and infertility has been previously assessed by several studies, with contradictory results ${ }^{(13,20)}$. Hence, we sought to perform a systematic review and meta-analysis of current evidence on this particular subject.

\section{METHODS}

\section{Criteria for study inclusion}

Meta-analysis was performed according to the recommendations of the Meta-analysis of Observational Studies in Epidemiology (MOOSE) Group ${ }^{(28)}$. Controlled studies with case-control or cohort study designs were considered for review. Uncontrolled observational studies and case series were excluded. Two kinds of studies were analyzed in-depth: those assessing the risk of infertility in subjects with previously diagnosed $\mathrm{CD}$, and those evaluating the prevalence of undiagnosed $\mathrm{CD}$ in subjects already diagnosed with infertility.

\section{Search strategy}

A computer-assisted search was performed from 1966 to December 2013 in the MEDLINE-Pubmed, EMBASE, LILACS and Cochrane Library databases. The search strategy consisted of the MESH terms "celiac disease" or "celiac sprue" and "infertility" or "genital diseases, female" or "sterility". There was no language restriction. We reviewed the bibliography of identified relevant papers. Additionally, abstracts from Digestive Disease Week and United European Gastroenterology Week 2008-2013 were manually reviewed.

Two authors independently performed the bibliographic search for potentially relevant studies.

Declared conflict of interest of all authors: none

Gastroenterology Division, Internal Medicine Department. Centro de Educación Médica e Investigación Clínica - CEMIC, Buenos Aires, Argentina

Correspondence: Juan Sebastian Lasa. 1958 Austria St. Buenos Aires, Argentina - ZIP Code 1425. Email: juanselasa@gmail.com 
Compatible abstracts were reviewed to check compliance with eligibility criteria. The findings of each author were then compared. If there was disagreement towards a particular study, its inclusion was decided by a third author. If there was evidence of duplicated data, original papers' authors were contacted in order to determine study inclusion or exclusion.

\section{Quality assessment}

Methodological quality of included studies was evaluated by two authors in an independent manner using the Newcastle-Ottawa assessment scale for case-control and cohort studies $^{(27)}$. If disagreement on a particular subject was encountered, a third author resolved the difference.

\section{Statistical analysis}

Meta-analysis was performed using REVMAN software (Review Manager Version 5.1. Copenhagen: The Nordic Cochrane Center, The Cochrane Collaboration, 2011). Heterogeneity was evaluated using the I 2 and chi square test. Because of anticipated variability in patient population and study design, a random-effect model was used. The first analysis included studies assessing the occurrence of non-diagnosed $\mathrm{CD}$ in patients suffering from infertility; the outcome was described as an Odds Ratio (OR) with a $95 \%$ confidence interval $(95 \% \mathrm{CI})$. In a second analysis, we included studies evaluating the odds of infertility in patients previously diagnosed with $\mathrm{CD}$. We also used an OR with a 95\% CI in order to describe the latter. Sensitivity analysis was performed according to heterogeneity or if significant methodological quality differences were found. Funnel plots were performed in order to assess potential publication bias.

\section{RESULTS}

The search identified 413 bibliographic citations. Of these, 395 were excluded, and eighteen potentially relevant studies were evaluated. Six studies were excluded because they did not fulfilled elegibility criteria: three studies lacked a control group ${ }^{(5,13,17)}$, a large population-based study ${ }^{(9)}$ was not designed to compare infertility prevalence in celiac subjects and used U.S. general population as a control group; another study ${ }^{(20)}$ used an inadequate control group (previously known prevalence of silent CD in the northen Sardinian population) and another one ${ }^{(7)}$ assessed the risk of infertility only in male subjects and used Crohn's disease patients as control group. Finally, as shown in Figure 1, twelve studies were included for analysis. Two of the analyzed studies were population-based cohort studies (Zugna 2010 and Tata 2005) which evaluated the risk of infertility in a CD population ${ }^{(29,31)}$. Two other studies (Molteni 1990 and Sher 1994) were case-control studies evaluating the odds of infertility in a CD group compared to a non-celiac control group ${ }^{(21,25)}$. Finally, eight studies (Collin 1996, Kohlo 1999, Shamaly 2004, de Silva Martins 2006, Tiboni 2006, Kumar 2010, Khoshbaten 2011, Hogen Esch 2011) assessed the occurrence of non-diagnosed CD in subjects suffering from infertility compared to apparently healthy controls $^{(4,6,12,14,15,16,24,30)}$. Two studies included data on male partners also evaluated for non-diagnosed $\mathrm{CD}$ versus controls (Khoshbaten 2011, Hogen Esch 2011).

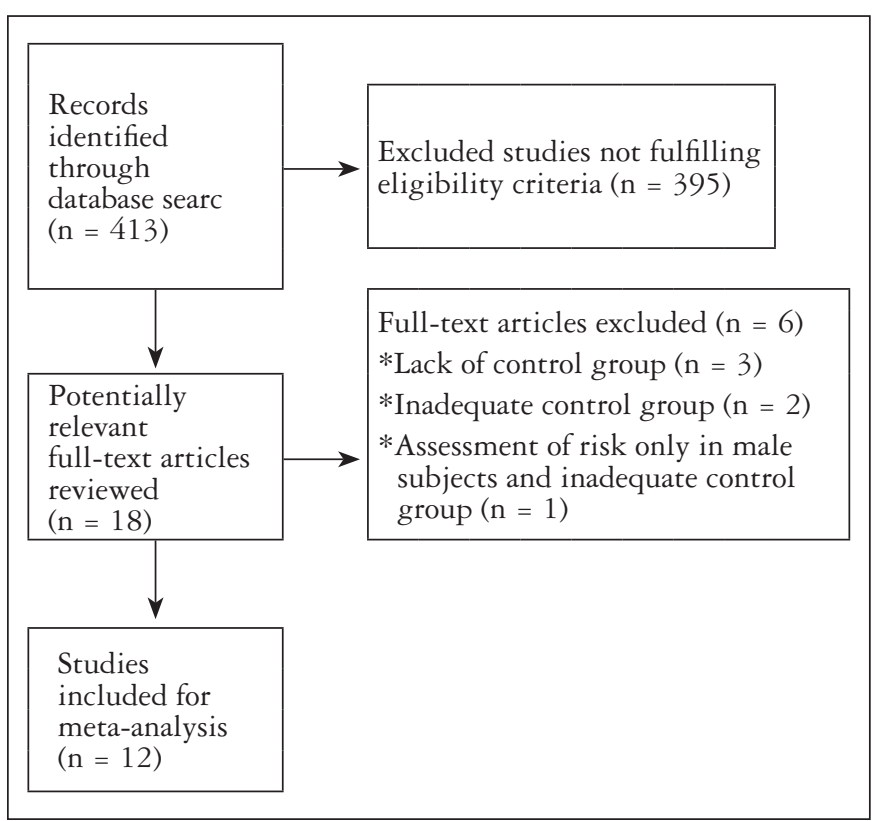

FIGURE 1. Flow chart outlining the study selection process

Characteristics of included studies are summarized in Table 1. Infertility was assessed in a similar fashion in most studies; however, this was not clearly stated in the studies by Moltoni et al., Sher et al., Zugna et al. and Tata et al. Three studies (Shamaly 2004, Kumar 2010 and Khoshbaten 2011) evaluated patients with unexplained infertility (UI) only.

Methodological assessment of included studies is described in Table 2. No study was excluded due to methodological limitations.

\section{Non-diagnosed celiac disease in subjects with infertility}

Meta-analytical findings of the first condition are described in Table 3. Overall, 4471 subjects were included in this analysis. No heterogeneity was observed (I2 $0 \% ; P 0.67)$, and a significant association was found between women with a diagnosis of infertility and CD [OR 3.09 (95\% CI 1.74-5.49)]. Sensitivity analysis was also performed; studies assessing all kinds of infertility (not only UI) were also excluded. No relevant differences were found as a result [OR3.41 (1.65-7.03)]. Further sensitivity analysis was carried out by excluding studies that did not use anti-transglutaminase and/or anti-endomysium antibodies for initial CD screening (Collin 1996); again, no significant difference was found in the result [OR 2.95 (1.64-5.31)].

Meta-analysis of studies which evaluated CD in male partners versus control subjects included 2054 patients and 
TABLE 1. Characteristics of included studies

\begin{tabular}{|c|c|c|c|c|c|c|}
\hline Author & Location & Subjects & Controls & $\begin{array}{l}\text { Type of } \\
\text { infertility } \\
\text { assessed }\end{array}$ & $\begin{array}{l}\text { Celiac disease } \\
\text { definition }\end{array}$ & Outcomes \\
\hline \multicolumn{7}{|c|}{ CASE-CONTROL AND CROSS SECTIONAL STUDIES } \\
\hline $\begin{array}{l}\text { Molteni } \\
\text { et al. }{ }^{(21)}\end{array}$ & Italy & $\begin{array}{l}38 \text { newly diagnosed } \\
\text { celiac disease women } \\
\text { with normal sexual life } \\
\text { without contraceptive } \\
\text { method }\end{array}$ & $\begin{array}{l}38 \text { women from hospital } \\
\text { staff or IBS outpatients, } \\
\text { matched for age, origin } \\
\text { and sexual behavior }\end{array}$ & $\begin{array}{l}\text { Not clearly } \\
\text { stated }\end{array}$ & $\begin{array}{l}\text { Jejunal biopsy and } \\
\text { GFD response }\end{array}$ & $\begin{array}{l}\text { Infertility in CD Group }=4 / 38 \\
(10.52 \%) \text {; Infertility in control } \\
\text { Group }=3 / 38(7.89 \%)\end{array}$ \\
\hline $\begin{array}{l}\text { Sher } \\
\text { et al. }{ }^{(24)}\end{array}$ & $\begin{array}{l}\text { United } \\
\text { Kingdom }\end{array}$ & $\begin{array}{l}68 \text { celiac disease } \\
\text { women from previously } \\
\text { performed epidemiologic } \\
\text { study }\end{array}$ & $\begin{array}{l}68 \text { sex and age- matched, } \\
\text { healthy individuals from } \\
\text { the same community } \\
\text { (one described source= } \\
\text { patients' relatives) }\end{array}$ & $\begin{array}{l}\text { Not clearly } \\
\text { stated }\end{array}$ & Not clearly stated & $\begin{array}{l}\text { Infertility in CD Group }=11 / 68 \\
(16.17 \%) ; \text { Infertility in control Group } \\
=1 / 68(1.47 \%) \text { (subject to recall bias) }\end{array}$ \\
\hline $\begin{array}{l}\text { Collin } \\
\text { et al. }{ }^{(4)}\end{array}$ & Finland & $\begin{array}{l}150 \text { women evaluated } \\
\text { for infertility; } 50 \text { women } \\
\text { with } \geqslant 2 \text { abortions [mean } \\
\text { age } 31(22-42) \text { ] }\end{array}$ & $\begin{array}{l}150 \text { women w/normal } \\
\text { obstetric history } \\
\text { undergoing sterilization }\end{array}$ & $\begin{array}{l}\text { All kind;98 } \\
(65.3 \%) \\
\text { unexplained } \\
\text { (UI) }\end{array}$ & $\begin{array}{l}\text { Screening w/IgA } \\
\text { AG+antireticulin; } \\
\text { confirmed by biopsy }\end{array}$ & $\begin{array}{l}\mathrm{CD} \text { in infertility Group }=4 / 150 \\
(2.7 \%) ; 4 / 4 \mathrm{UI}(4.1 \%) ; \mathrm{CD} \text { in control } \\
\text { Group }=0 / 150\end{array}$ \\
\hline $\begin{array}{l}\text { Kohlo } \\
\text { et al. } .^{(15)}\end{array}$ & Finland & $\begin{array}{l}129 \text { women evaluated } \\
\text { at the department } \\
\text { of obstetrics and } \\
\text { gynecology }\end{array}$ & $\begin{array}{l}51 \text { apparently healthy } \\
\text { women working at } \\
\text { hospital }\end{array}$ & $\begin{array}{l}\text { All kind; } 47 \\
(36.43 \%) \mathrm{UI}\end{array}$ & $\begin{array}{l}\text { IgA AE (not } \\
\text { confirmed by } \\
\text { biopsy) }\end{array}$ & $\begin{array}{l}\mathrm{CD} \text { in infertility Group }=1 / 129 \\
(0.77 \%) ; 1 / 1 \text { UI } \quad(1 / 47=2.12 \%) ; \mathrm{CD} \\
\text { in control Group }=1 / 51(1.96 \%)\end{array}$ \\
\hline $\begin{array}{l}\text { Shamaly } \\
\text { et al. }{ }^{(23)}\end{array}$ & Israel & $\begin{array}{l}192 \text { women attending } \\
\text { infertility clinic (age } \\
\text { range 18-44) }\end{array}$ & $\begin{array}{l}210 \text { women attending } \\
\text { pediatric clinic (age range } \\
18-44 \text { ) }\end{array}$ & UI & $\begin{array}{l}\text { IgA AtTG; IgA } \\
\text { AE; confirmation by } \\
\text { biopsy }\end{array}$ & $\begin{array}{l}\text { CD in infertility Group }=5 / 192 \\
(2.6 \%) ; C D \text { in control } \\
\text { Group }=1 / 210(0.48 \%)\end{array}$ \\
\hline $\begin{array}{l}\text { Tiboni } \\
\text { et al. }{ }^{(29)}\end{array}$ & Italy & $\begin{array}{l}200 \text { women undergoing } \\
\text { ART (mean age } 35 \pm 5 \text { ) }\end{array}$ & $\begin{array}{l}200 \text { apparent healthy } \\
\text { women with at least } 1 \\
\text { child }\end{array}$ & $\begin{array}{l}\text { All kind; } \\
26.5 \% \text { UI }\end{array}$ & $\begin{array}{l}\text { Screening w/IgA } \\
\text { AtTG+ IgA AE; } \\
\text { confirmation by } \\
\text { biopsy }\end{array}$ & $\begin{array}{l}\text { CD in infertility Group }=5 / 200 \\
(2.5 \%) ; 0 / 5 \text { UI. CD in control Group } \\
=2 / 200(1 \%)\end{array}$ \\
\hline $\begin{array}{l}\text { da Silva } \\
\text { Martins } \\
\text { et al. }{ }^{(6)}\end{array}$ & Brazil & $\begin{array}{l}200 \text { women attending } \\
\text { hospital due to infertility }\end{array}$ & $\begin{array}{l}400 \text { women attending } \\
\text { hospital for other reason } \\
\text { than infertility }\end{array}$ & All kind & $\begin{array}{l}\text { Screening w/ IgA } \\
\text { AE; confirmation by } \\
\text { biopsy }\end{array}$ & $\begin{array}{l}\mathrm{CD} \text { in infertility Group }=3 / 200 \\
(1.5 \%) ; \mathrm{CD} \text { in control Group }=0 / 400\end{array}$ \\
\hline $\begin{array}{l}\text { Kumar } \\
\text { et al. }{ }^{(16)}\end{array}$ & India & $\begin{array}{l}230 \text { women attending } \\
\text { infertility clinic (mean } \\
\text { age } 29.7 \pm 4 \text { ) }\end{array}$ & $\begin{array}{l}305 \text { women w/ normal } \\
\text { obstetric history }\end{array}$ & UI & $\begin{array}{l}\operatorname{IgA} / \operatorname{IgG} A G ; \operatorname{Ig} A \\
A t T G+\operatorname{IgA~AE}\end{array}$ & $\begin{array}{l}\text { CD in infertility Group }=13 / 230 \\
(5.6 \%) ; \mathrm{CD} \text { in control } \\
\text { Group }=4 / 305(1.3 \%)\end{array}$ \\
\hline $\begin{array}{l}\text { Khoshbaten } \\
\text { et al. }{ }^{(14)}\end{array}$ & Azerbaijan & $\begin{array}{l}100 \text { women attending } \\
\text { infertility clinic (w/ } \\
\text { male Partners) (mean age } \\
29 \pm 6)\end{array}$ & $\begin{array}{l}200 \text { apparently healthy } \\
\text { couples with at least } 1 \\
\text { birth }\end{array}$ & UI & $\begin{array}{l}\text { IgA AtTG } \\
\text { (confirmed by } \\
\text { biopsy) }\end{array}$ & $\begin{array}{l}\mathrm{CD} \text { in infertility Group }=8 / 100(8 \%) ; \\
\mathrm{CD} \text { in control Group }=7 / 200(3.5 \%) ; \\
\mathrm{CD} \text { in male } \\
\text { Partners }=5 / 100(5 \%) ; \mathrm{CD} \text { in male } \\
\text { controls }=4 / 200(2 \%)\end{array}$ \\
\hline $\begin{array}{l}\text { Hogen } \\
\text { Ersch } \\
\text { et al. }{ }^{(12)}\end{array}$ & Netherlands & $\begin{array}{l}1038 \text { women attending } \\
\text { to infertility clinic (mean } \\
\text { age } 32.3 \text {; range } 20-45 \text { ) } \\
\text { (w/male Partners) }\end{array}$ & $\begin{array}{l}1432 \text { healthy community } \\
\text { adults ( } 716 \text { female) }\end{array}$ & $\begin{array}{l}\text { All kind; 34\% } \\
\text { UI }\end{array}$ & $\begin{array}{l}\text { IgA AtTG; IgA } \\
\text { AE (endoscopy not } \\
\text { offered) }\end{array}$ & $\begin{array}{l}\text { CD in infertility Group }=6 / 1038 \\
(0.58 \%) ; \mathrm{CD} \text { in UI }=2 / 351(0.57 \%) ; \\
\mathrm{CD} \text { in control. } \\
\text { Group }=2 / 716(0.28 \%) ; \\
\mathrm{CD} \text { in male Partners }=4 / 1038(0.39 \%) ; \\
\mathrm{CD} \text { in male controls }=3 / 716(0.42 \%)\end{array}$ \\
\hline \multicolumn{7}{|c|}{ COHORT STUDIES } \\
\hline $\begin{array}{l}\text { Tata } \\
\text { et al. }{ }^{(28)}\end{array}$ & $\begin{array}{l}\text { United } \\
\text { Kingdom }\end{array}$ & $\begin{array}{l}1521 \text { celiac women in } \\
\text { fertile age }(18-44 \text { y) } \\
\text { between } 1987-2002\end{array}$ & $\begin{array}{l}7732 \text { women without } \\
\text { CD matched by age, } \\
\text { sex, general practice and } \\
\text { follow-up time }\end{array}$ & $\begin{array}{l}\text { Not } \\
\text { applicable }\end{array}$ & $\begin{array}{l}\text { Records from } \\
\text { GPRD (criteria not } \\
\text { specified) }\end{array}$ & $\begin{array}{l}\text { Births in CD Group = } 341 \text { (median } \\
\text { follow-up }=4.3 y) ; \text { births in control } \\
\text { Group = } 1903 \text { (median follow-up = } \\
4.9 y) ; \text { HR } 1.01(95 \% \text { CI0.91-1.14) }\end{array}$ \\
\hline $\begin{array}{l}\text { Zugna } \\
\text { et al. } .^{(30)}\end{array}$ & Sweden & $\begin{array}{l}11495 \text { celiac women } \\
\text { born between } 1920-1990\end{array}$ & $\begin{array}{l}51109 \text { women without } \\
\text { CD matched by age, } \\
\text { sex, calendar period and } \\
\text { county of residence }\end{array}$ & $\begin{array}{l}\text { Not } \\
\text { applicable }\end{array}$ & $\begin{array}{l}\text { Swedish regional } \\
\text { biopsy data }\end{array}$ & $\begin{array}{l}\text { Births in CD Group = 6870; } \\
\text { HR } 1.04(95 \% \text { CI 1.01-1.07) }\end{array}$ \\
\hline
\end{tabular}

IBS: Irritable bowel syndrome, AE: Anti-endomysium, GFD: Gluten-free diet, AtTG: Anti-transglutaminase, CD: Celiac disease, HLA: Human leukocyte antigen, AG: Anti-gliadin, GPRD: General practice research database, UI: Unexplained infertility, HR: Hazard ratio 
TABLE 2. Methodological characteristics of included studies

\begin{tabular}{|c|c|c|c|c|c|c|c|c|}
\hline \multicolumn{9}{|c|}{ CASE-CONTROL AND CROSS_SECTIONAL STUDIES } \\
\hline Author & $\begin{array}{l}\text { Case } \\
\text { definition } \\
\text { adequate? }\end{array}$ & $\begin{array}{l}\text { Representa- } \\
\text { tiveness of } \\
\text { cases }\end{array}$ & $\begin{array}{l}\text { Selection of } \\
\text { controls }\end{array}$ & $\begin{array}{l}\text { Definition of } \\
\text { controls }\end{array}$ & $\begin{array}{l}\text { Comparability } \\
\text { of cases and } \\
\text { controls }\end{array}$ & $\begin{array}{l}\text { Ascertainment } \\
\text { of exposure }\end{array}$ & $\begin{array}{l}\text { Same } \\
\text { method of } \\
\text { ascertainment }\end{array}$ & $\begin{array}{l}\text { Non-response } \\
\text { rate }\end{array}$ \\
\hline $\begin{array}{l}\text { Molteni } \\
\text { et al. }\end{array}$ & Yes & $\begin{array}{l}\text { Consecutive } \\
\text { series }\end{array}$ & $\begin{array}{l}\text { Hospital and } \\
\text { community } \\
\text { controls }\end{array}$ & $\begin{array}{l}\text { No history of } \\
\text { disease }\end{array}$ & $\begin{array}{l}\text { Sex, age and } \\
\text { origin adjustment }\end{array}$ & Questionnaire & Yes & Not stated \\
\hline $\begin{array}{l}\text { Sher } \\
\text { et al. }{ }^{(24)}\end{array}$ & Not clear & $\begin{array}{l}\text { Consecutive } \\
\text { series }\end{array}$ & $\begin{array}{l}\text { Community } \\
\text { (cases' friends } \\
\text { or relatives } \\
\text { enrolled) }\end{array}$ & $\begin{array}{l}\text { No history of } \\
\text { disease }\end{array}$ & $\begin{array}{l}\text { Sex and age } \\
\text { adjustment }\end{array}$ & Questionnaire & Yes & $\begin{array}{l}67 \% \text { response rate } \\
\text { in cases; } 66 \% \text { in } \\
\text { controls }\end{array}$ \\
\hline $\begin{array}{l}\text { Collin } \\
\text { et al. }{ }^{(4)}\end{array}$ & Yes & $\begin{array}{l}\text { Consecutive } \\
\text { series }\end{array}$ & $\begin{array}{l}\text { Hospital } \\
\text { controls }\end{array}$ & $\begin{array}{l}\text { No history of } \\
\text { disease }\end{array}$ & No adjustment & $\begin{array}{l}\text { Laboratory+ } \\
\text { biopsy when }+ \\
\text { ve screening }\end{array}$ & Yes & Not stated \\
\hline $\begin{array}{l}\text { Kohlo } \\
\text { et al. }{ }^{(15)}\end{array}$ & Yes & $\begin{array}{l}\text { Consecutive } \\
\text { series }\end{array}$ & $\begin{array}{l}\text { Hospital } \\
\text { controls }\end{array}$ & $\begin{array}{l}\text { No history of } \\
\text { disease }\end{array}$ & No adjustment & Laboratory & Yes & Not stated \\
\hline $\begin{array}{l}\text { Shamaly } \\
\text { et al. }{ }^{(23)}\end{array}$ & Yes & $\begin{array}{l}\text { Consecutive } \\
\text { series }\end{array}$ & $\begin{array}{l}\text { Hospital } \\
\text { controls }\end{array}$ & $\begin{array}{l}\text { No history of } \\
\text { disease }\end{array}$ & $\begin{array}{l}\text { Age and sex } \\
\text { adjustment }\end{array}$ & $\begin{array}{l}\text { Laboratory+ } \\
\text { biopsy when }+ \\
\text { ve screening }\end{array}$ & Yes & Not stated \\
\hline $\begin{array}{l}\text { Tiboni } \\
\text { et al. }{ }^{(29)}\end{array}$ & Yes & $\begin{array}{l}\text { Consecutive } \\
\text { series }\end{array}$ & $\begin{array}{l}\text { Hospital } \\
\text { controls }\end{array}$ & $\begin{array}{l}\text { No history of } \\
\text { disease }\end{array}$ & No adjustment & $\begin{array}{l}\text { Laboratory+ } \\
\text { biopsy when + } \\
\text { ve screening }\end{array}$ & Yes & Not stated \\
\hline $\begin{array}{l}\text { da Silva Martins } \\
\text { et al. }{ }^{(6)}\end{array}$ & Yes & $\begin{array}{l}\text { Consecutive } \\
\text { series }\end{array}$ & $\begin{array}{l}\text { Hospital } \\
\text { controls }\end{array}$ & $\begin{array}{l}\text { No history of } \\
\text { disease }\end{array}$ & $\begin{array}{l}\text { Age and sex } \\
\text { adjustment }\end{array}$ & $\begin{array}{l}\text { Laboratory }+ \\
\text { biopsy when }+ \\
\text { ve screening } \\
\end{array}$ & Yes & Not stated \\
\hline $\begin{array}{l}\text { Kumar } \\
\text { et al. }{ }^{(16)}\end{array}$ & Yes & $\begin{array}{l}\text { Consecutive } \\
\text { series }\end{array}$ & $\begin{array}{l}\text { Hospital } \\
\text { controls }\end{array}$ & $\begin{array}{l}\text { No history of } \\
\text { disease }\end{array}$ & No adjustment & Laboratory & Yes & Not stated \\
\hline $\begin{array}{l}\text { Khoshbaten } \\
\text { et al. }{ }^{(14)}\end{array}$ & Yes & $\begin{array}{l}\text { Consecutive } \\
\text { series }\end{array}$ & $\begin{array}{l}\text { Hospital } \\
\text { controls }\end{array}$ & $\begin{array}{l}\text { No history of } \\
\text { disease }\end{array}$ & No adjustment & $\begin{array}{l}\text { Laboratory+ } \\
\text { biopsy when }+ \\
\text { ve screening } \\
\end{array}$ & Yes & Not stated \\
\hline $\begin{array}{l}\text { Hogen Ersch } \\
\text { et al. }{ }^{(12)}\end{array}$ & Yes & $\begin{array}{l}\text { Consecutive } \\
\text { series }\end{array}$ & Community & Not stated & No adjustment & Laboratory & Yes & Not stated \\
\hline \multicolumn{9}{|c|}{ COHORT STUDIES } \\
\hline & $\begin{array}{l}\text { Represen- } \\
\text { tativeness } \\
\text { of exposed } \\
\text { cohort }\end{array}$ & $\begin{array}{l}\text { Unexposed } \\
\text { cohort }\end{array}$ & $\begin{array}{l}\text { Ascertainment } \\
\text { of exposure }\end{array}$ & $\begin{array}{l}\text { Demonstration that } \\
\text { outcome was not } \\
\text { present at the start } \\
\text { of study }\end{array}$ & $\begin{array}{l}\text { Comparability of } \\
\text { cohorts }\end{array}$ & $\begin{array}{l}\text { Assessment of } \\
\text { outcome }\end{array}$ & $\begin{array}{l}\text { Was follow-up } \\
\text { long enough? }\end{array}$ & $\begin{array}{l}\text { Adequacy of } \\
\text { follow-up }\end{array}$ \\
\hline $\begin{array}{l}\text { Tata } \\
\text { et al. }{ }^{(28)}\end{array}$ & $\begin{array}{l}\text { Representa- } \\
\text { tive }\end{array}$ & $\begin{array}{l}\text { Same } \\
\text { community }\end{array}$ & $\begin{array}{l}\text { Not clear if } \\
\text { biopsy was } \\
\text { required }\end{array}$ & Yes & $\begin{array}{l}\text { Adjusted for } \\
\text { age, sex, general } \\
\text { practice and } \\
\text { follow-up time }\end{array}$ & Record linkage & Yes & Not applicable \\
\hline $\begin{array}{l}\text { Zugna } \\
\text { et al. }{ }^{(30)}\end{array}$ & $\begin{array}{l}\text { Representa- } \\
\text { tive }\end{array}$ & $\begin{array}{l}\text { Same } \\
\text { community }\end{array}$ & $\begin{array}{l}\text { Adequate } \\
\text { (biopsy } \\
\text { database) }\end{array}$ & Yes & $\begin{array}{l}\text { Adjusted for age, } \\
\text { sex, calendar } \\
\text { period and county } \\
\text { of residence }\end{array}$ & Record linkage & Yes & Not applicable \\
\hline
\end{tabular}

TABLE 3. Meta-analysis of case-control studies assessing frequency of undiagnosed celiac disease in infertility patients

\begin{tabular}{|c|c|c|c|c|c|c|c|c|c|}
\hline \multirow[b]{2}{*}{ Study or Subgroup } & \multicolumn{2}{|c|}{ Infertility } & \multicolumn{2}{|c|}{ Control } & \multicolumn{3}{|c|}{ Odds Ratio } & \multirow{2}{*}{\multicolumn{2}{|c|}{$\begin{array}{c}\text { Odds Ratio } \\
\text { M-H, Random, 95\% CI }\end{array}$}} \\
\hline & Events & Total & Events & Total & Weight & M-H, Random, 95\% CI & Year & & \\
\hline Collin et $\mathrm{al}^{(4)}$ & 4 & 150 & 0 & 150 & $3.9 \%$ & $9.25[0.49,173.25]$ & 1996 & & \\
\hline Kohlo et al. ${ }^{(15)}$ & 1 & 129 & 1 & 51 & $4.2 \%$ & $0.39[0.02,6.37]$ & 1999 & & 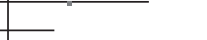 \\
\hline Shamaly et al. ${ }^{(23)}$ & 5 & 192 & 1 & 210 & $7.1 \%$ & $5.59[0.65,48.27]$ & 2004 & & \\
\hline da Silva Martins et al. ${ }^{(6)}$ & 3 & 200 & 0 & 400 & $3.8 \%$ & $14.19[0.73,276.16]$ & 2006 & & \\
\hline Tiboni et al. ${ }^{(29)}$ & 5 & 200 & 2 & 200 & $12.1 \%$ & $2.54[0.49,13.24]$ & 2006 & & F- \\
\hline Kumar et al. ${ }^{(16)}$ & 13 & 230 & 4 & 305 & $25.7 \%$ & $4.51[1.45,14.01]$ & 2010 & & $\longrightarrow$ \\
\hline Hogen Esch et al. ${ }^{(12)}$ & 6 & 1038 & 2 & 716 & $12.9 \%$ & $2.08[0.42,10.31]$ & 2011 & & \\
\hline Khoshbaten et al. ${ }^{(14)}$ & 8 & 100 & 7 & 200 & $30.3 \%$ & $2.40[0.84,6.81]$ & 2011 & & $f-$ \\
\hline Total $(95 \% \mathrm{CI})$ & & 2239 & & 2232 & $100.0 \%$ & $3.09[1.74,5.49]$ & & & $>$ \\
\hline Total events & 45 & & 17 & & & & & & \\
\hline \multicolumn{8}{|c|}{$\begin{array}{l}\text { Heterogeneity: } \mathrm{Tau}^{2}=0.00 ; \mathrm{Chi}^{2}=4.95, \mathrm{df}=7(P=0.67) ; \mathrm{I}^{2}=0 \% \\
\text { Test for overall effect: } \mathrm{Z}=3.84(P=0.0001)\end{array}$} & $\begin{array}{ll}0.001 & 0.1 \\
\text { Favours } & \text { Control }\end{array}$ & $\begin{array}{l}10 \\
10 \\
\text { Favours Infertility }\end{array}$ \\
\hline
\end{tabular}


did not show a significant association [OR 1.63 (95\% CI 0.64.46)], as described in Table 4. Figure 2A shows the funnel plot of included studies mentioned above, with no significant asymmetry (Egger test, $P=0.3$ ).

\section{Infertility in subjects with diagnosed celiac disease}

When considering studies assessing the occurrence of infertility in subjects with CD, 33636 subjects were included for analysis. No difference was found between CD patients and control subjects [OR $0.99(95 \%$ CI 0.86-1.13)], as described in Table 5. Significant heterogeneity was corrected by the exclusion of one of the studies (Sher 1994), which did not alter the result of the analysis [OR 0.97 (0.92-1.03)]. Figure 2B shows funnel plot of these studies, with an asymmetry that could be related to a publication bias (Egger test, $P<0.001$ ).

\section{DISCUSSION}

Our meta-analysis evaluates the association between a frequent reproductive disorder such as infertility and $\mathrm{CD}$.
According to our results, non-diagnosed untreated CD constitutes a risk factor significantly associated with infertility in women. When comparing studies that enrolled patients previously diagnosed with $\mathrm{CD}$, this association is not as evident as in the former context. This could be related to the effect that adoption of a gluten-free diet (GFD) may have on this particular health issue.

The association between $\mathrm{CD}$ and female reproductive disorders has been previously described ${ }^{(3)}$. It is well known that CD increases the risk of recurrent miscarriages, low weight at birth, early menopause and other pregnancy disorders $^{(10,11,18,19,26)}$. It is also known that these kinds of complications appear to diminish once a GFD is implemented.

Infertility is defined as the impossibility of conceiving after 12 months of unprotected intercourse ${ }^{(1)}$. It can be related to both male and female factors, with the latter subdivided into ovarian, tubal, endometrial or unexplained causes. It is relatively frequent, with approximately $8 \%-12 \%$ of couples with women at child-bearing age carrying this condition $^{(2)}$

TABLE 4. Meta-analysis of case-control studies assessing frequency of undiagnosed celiac disease in the male partners of infertility patients

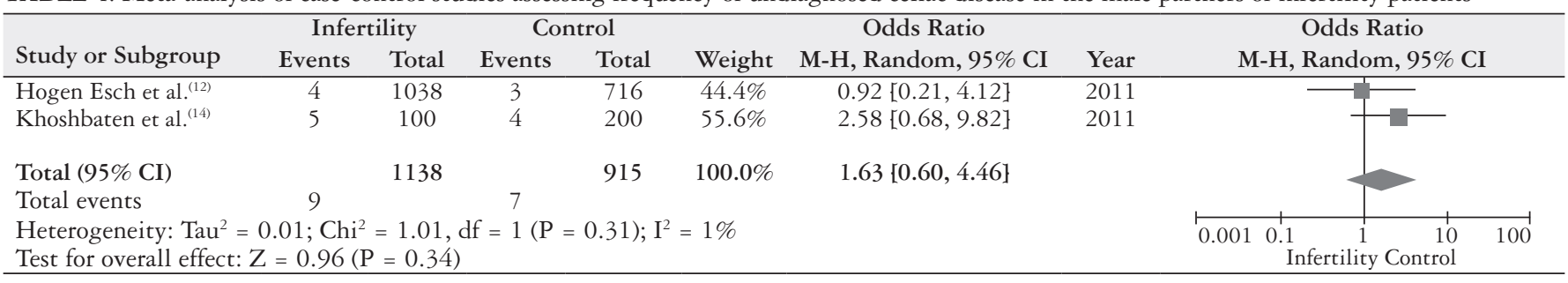

TABLE 5. Meta-analysis of case-control studies assessing frequency of undiagnosed celiac disease in infertility patients

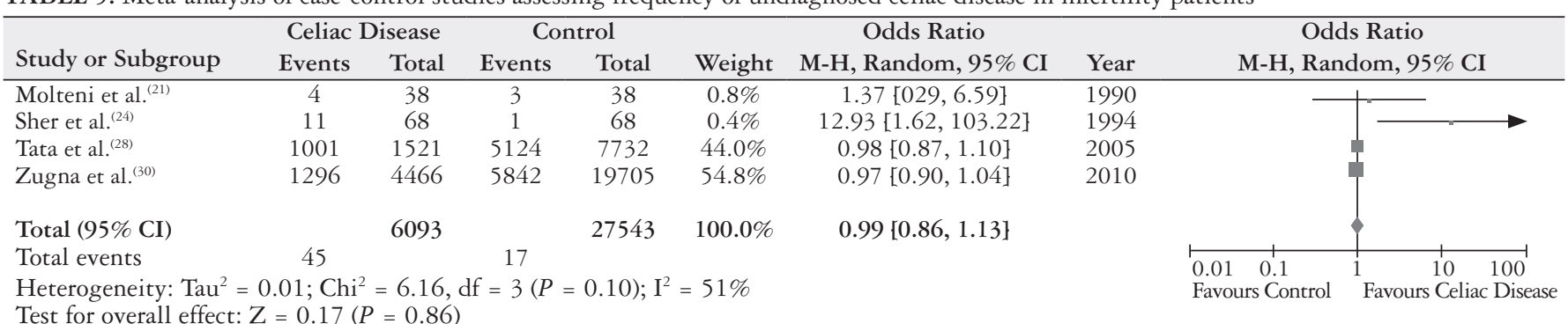

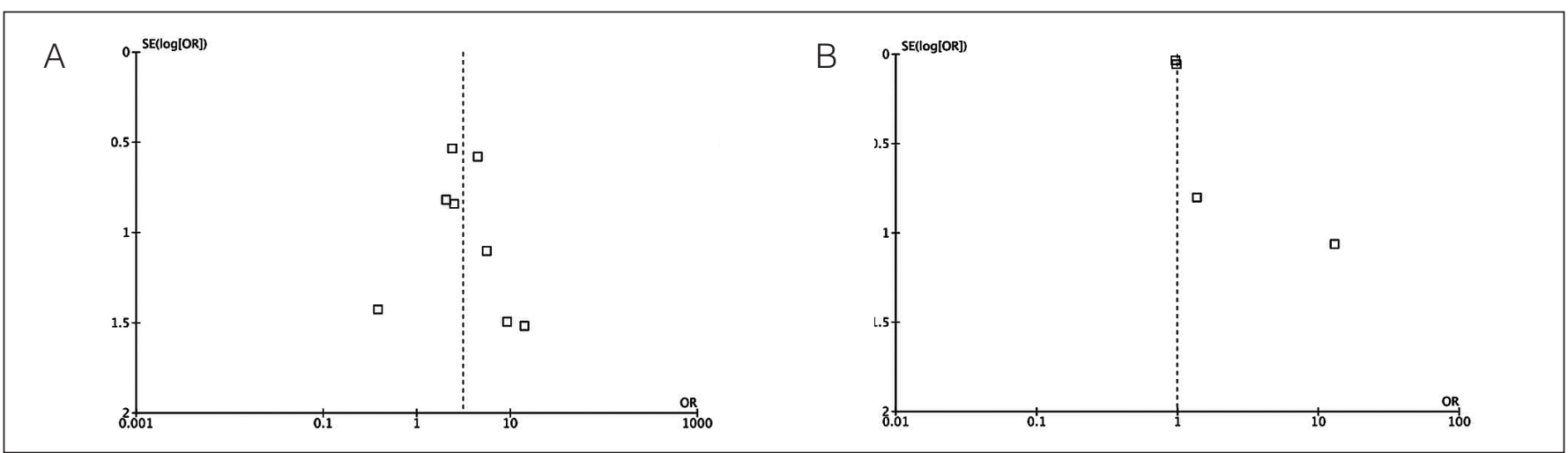

FIGURE 2. Funnel plot assessing for publication bias among (A) studies assessing undiagnosed celiac disease risk in patients with infertility. (B) studies assessing risk of infertility in already-diagnosed celiac disease 
Reports on infertility risk in CD patients are far from scarce, but often show non-definite, sometimes conflicting results. Jackson et al. ${ }^{(13)}$ described a cohort of women suffering from infertility that were tested for CD by means of serology. This study found a relatively low frequency of $\mathrm{CD}$ among infertile women compared to the background rate in the general U.S. population. Choi et al. ${ }^{(5)}$ tested women assisting at an infertility clinic for CD; although an increased prevalence overall was not found, $\mathrm{CD}$ was more frequent in those women with UI; again, this study lacked a control group.

Case-control and cross-sectional studies assessing the risk of $\mathrm{CD}$ in infertile patients also show conflicting results. The largest study published by Hogen Esch et al. ${ }^{(12)}$ included 1038 patients attending to an infertility clinic and 1432 controls. They did not find a significant difference in CD prevalence between groups; it is worth mentioning though that they only performed serological tests to determine $\mathrm{CD}$ prevalence. Da Silva Martins et al. ${ }^{(6)}$ published the only experience so far in a South-American setting: they included 200 patients attending an infertility clinic and 400 control subjects. They also were not able to find a significant $\mathrm{CD}$ prevalence among infertile women.

Only the study by Kumar et al. ${ }^{(16)}$ proved a significant association between $\mathrm{CD}$ and infertility. The other studies included in this analysis did not reach a statistically relevant difference, probably due to insufficient sample size. However, as revealed in our meta-analysis, the pooled OR is significantly increased.

These results contrast with those derived from cohort and case-control studies including previously diagnosed CD subjects. Tata et al. ${ }^{(29)}$ published a large cohort study that assessed $1521 \mathrm{CD}$ women in fertile age and 7732 non-CD women matched by age, sex, general practice and follow-up time. They found that the birth rates in both groups with a similar median follow-up time (4.3 vs 4.9 years) were similar (HR 1.01 95\%CI 0.91-1.14). What is more, Zugna et al. ${ }^{(31)}$ published an even larger cohort study including 11495 women with CD and 51109 controls: they also did not find en excessive risk of infertility in the CD cohort. These results contrast with those from studies that included non-diagnosed CD. A potential explanation for this difference may be that in the cohort studies, CD patients were already treated: this may underline that GFD may cause a reversal of infertility problems in celiac women. Cohort studies including alreadydiagnosed CD women did not assess GFD compliance, so it is not possible to conclude that gluten ingestion may actually be a risk factor for developing infertility in CD subjects. However, Zugna et al. ${ }^{(31)}$ found that although women with $\mathrm{CD}$ had normal fertility overall, fertility levels decreased in the last two years preceding $\mathrm{CD}$ diagnosis. This emphasizes the fact that untreated CD is a risk factor for infertility and that GFD may be sufficient to correct this abnormality.

There is no clear explanation why these two entities are associated. CD is associated with many extra-intestinal diseases that have an immunological basis. Persistent inflammation can produce some serological markers that may have an impact in the development of infertility ${ }^{(22)}$. As seen with other extra-intestinal diseases, early introduction of a GFD may decrease the risk of this complication. Further studies should focus on this particular subject.

Although evidence is relatively scarce, studies evaluating male partners failed to show a significant association between infertility and CD. However, these studies do not mention a relationship between $\mathrm{CD}$ and certain factors related to male infertility, such as hypogonadism or semen quality deficiencies. Farthing et al.(7) described a high frequency of seminal alterations and sexual dysfunction in a cohort of $\mathrm{CD}$ male patients compared to Crohn's disease subjects.

Limitations must be taken into consideration. Among all of the studies analyzed, heterogeneity in CD definition and serological tests used for screening were found. Most of them, however, required histological confirmation. Secondly, the impact that GFD may exert cannot be estimated due to the design of included studies. Furthermore, the impact of poor adherence to GFD cannot be calculated from available evidence. Although funnel plot analysis doesn't suggest a significant publication bias when considering studies assessing CD risk in subjects with infertility, there is a significant asymmetry in the funnel plot of studies assessing risk of infertility in already-diagnosed CD patients.

In conclusion, this meta-analysis shows that undiagnosed $\mathrm{CD}$ is in fact a risk factor for infertility. This may have a significant impact on the management of infertility, since women seeking medical advice for this particular condition should be screened for CD. Ultimately, adoption of a GFD could have a positive impact on fertility in this group of patients. 
Lasa JS, Zubiaurre I, Soifer LO. Risco de infertilidade em pacientes com doença celíaca: uma meta-análise de estudos observacionais. Arq Gastroenterol. 2014,51(2):144-50.

RESUMO - Contexto - A doença celíaca é uma doença auto imune do intestino delgado associada a manifestações extra-intestinais, tais como distúrbios reprodutivos. A relação entre a doença celíaca e a infertilidade foi previamente avaliada, com resultados conflitantes. Objetivos - Procuramos determinar a relação entre a doença celíaca e a infertilidade. Método - Utilizou-se dados extraídos de estudos de projeto de caso-controle ou coorte de 1966 à dezembro de 2013 utilizando o MEDLINE-Pubmed, EMBASE, LILACS e Cochrane Library. Foram analisados dois tipos de ensaios: os que avaliam o risco de infertilidade em pacientes com doença celíaca já diagnosticados e, os que avaliam a prevalência da doença celíaca não diagnosticada em indivíduos com diagnóstico de infertilidade. Resultados - A busca resultou em 413 estudos potencialmente relevantes para a revisão, 12 dos quais foram finalmente incluídos para análise. Foi encontrada uma associação significativa entre as mulheres com diagnóstico de infertilidade e doença celíaca não diagnosticada [TR 3,09 (IC 95\% 1,74-5,49)]. Ao considerar esses estudos avaliando a ocorrência de infertilidade em pacientes com doença celíaca já diagnosticada, não foi encontrada diferença entre os pacientes com doença celíaca e controles [TR 0,99 (0,86-1,13)]. Conclusão-A falta de diagnóstico para a doença celíaca é um fator de risco para a infertilidade. As mulheres que procuram o conselho médico para esta condição particular, devem ser examinados para a doença celíaca. A adoção de uma dieta sem glúten pode ter um impacto positivo sobre a fertilidade neste grupo de pacientes.

DESCRITORES - Doença celíaca. Infertilidade feminina. Dieta livre de glúten.

\section{REFERENCES}

1. Balen AH, Rutherford AJ. Management of infertility. BMJ 2007;335:608-11.

2. Boiving J, Bunting L, Collins JA, Nygren KG. International estimates of infertility prevalence and treatment seeking: potential need and demandfor infertility medical care. Hum Reprod. 2007;22:1506-12.

3. Ciacci C, Cirillo M, Auriemma G, Di Dato G, Sabatini F, Mazzacca G. Celiac disease and pregnancy outcome. Am J Gastroenterol. 1996;91:718-22.

4. Collin P, Vilska S, Heinonen PK, Hallstrom O, Pikkarainen P. Infertility and coeliac disease. Gut. 1996;39:382-4.

5. Choi JM, Lebwohl B, Wang J, Lee SK, Murray JA, Sauer MV, Green PH. Increased prevalence of celiac diseasein patients with unexplained infertility in the United States: a prospective study. J Reprod Med. 2011;56:199-203.

6. Da Silva Martins CL, Gandolfi L, Tauil PL, de Almeida Rocha Picanco M, Galvao de Araujo MO, Pratesi R. Celiac disease and female infertility: a frequently neglected association. Rev Bras Ginecol Obstet. 2006;28:601-6.

7. Farthing MJG, Edwards CRW, Rees LH, Dawson AM: Male gonadal function in coeliac disease: sexual dysfunction, infertility and semen quality. Gut 1982; 23 : 608-614.

8. Fasano A, Catassi C. Clinical practice. Celiac disease. N Engl J Med. 2012; 367:2419-26.

9. Fasano A, Berti I, Gerarduzzi T, Not T, Colletti RB, Drago S, et al. Prevalence of celiac diseasein at-risk and not-at-risk groups in the United States. Arch Intern Med. 2003;163:286-92.

10. Gasbarrini A, Torre ES, Trivellini C, De Carolis S, Caruso A, Gasbarrini G Recurrent spontaneous abortionand intrauterine fetal growth retardation as symptoms of coeliac disease. Lancet. 2000;356:399-400.

11. Greco L, Veneziano A, Di Donato L, Zampella C, Pecoraro M, Paladini D, et al Undiagnosed coeliac disease does not appear to be associated with unfavourable outcome of pregnancy. Gut. 2004;53:149-51.

12. Hogen Esch CE, Van Rijssen MJL, Roos A, Koning F, Dekker FW, Mearin ML, et al. Screening for unrecognized coeliac disease in subfertile couples. Scand J Gastroenterol. 2011;46:1423-8.

13. Jackson JE, Rosen M, Mc Lean T, Moro J, Croughan M, Cedars MI. Prevalence of celiac disease in a cohort of women with unexplained infertility. Fertil Steril. 2008;89:1002-4.

14. Khoshbaten M, Nejad MR, Farzady L, Sharifi N, Hashemi SH, Rostami K. Fertility disorder associated with celiac disease in males and females: fact or fiction? J Obstet Gynael Res. 2011;37:1308-12.

15. Kolho KL, Tiitinen A, Tulppala M, Unkila-Kallio L, Savilahti E. Screening for coeliac disease in women with a history of recurrent miscarriage or infertility. $\mathrm{Br}$ J Obst Gynaecol. 1999;106:171-3.
16. Kumar A, Meena M, Begum N, Kumar N, Gupta RK, Agarwal S, et al. Latent celiac disease in reproductive perfomance of women. Fertil Steril. 2011;95:922-7.

17. Machado AP, Silva LR, Zausner B, Oliveira J de A, Diniz DR, de Oliveira J. Undiagnosed celiac disease in women with infertility. J Reprod Med. 2013;58:61-6.

18. Martinelli D, Fortunato F, Tafuri S, Germinario CA, Prato R. Reproductive life disorders in italian celiac women. A case-control study. BMC Gastroenterology. 2010;10:89.

19. Martinelli P, Troncone R, Paparo F, Torre P, Trapanese E, Fasano C, et al. Coeliac disease and unfavourable outcome of pregnancy. Gut. 2000;46:332-5.

20. Meloni GF, Dessole S, Vargiu N, Tomasi PA, Musumeci S. The prevalence of coeliac disease in infertility. Hum Reprod. 1999;14:2759-61.

21. Molteni N, Bardella MT, Bianchi PA. Obstetric and gynecological problems in women with untreated celiac sprue. J Clin Gastroenterol. 1990;12:37-9.

22. Östensson M, Montén C, Bacelis J, Gudjonsdottir AH, Adamovic S, Ek J, et al. Presentation of celiac disease. Gastrointest Endosc Clin N Am. 2012;22:613-21.

23. Reilly NR, Fasano A, Green PH. Presentation of celiac disease. Gastrointest Endosc Clin N Am. 2012;22:613-21.

24. Shamaly H, Mahameed A, Sharony A, Shamir R. Infertility and celiac disease: do we need more than one serological marker? Acta Obstet Gynecol Scand. 2004;83:1184-8.

25. Sher KS, Mayberry JF. Female fertility, obstetric and gynaecological history in coeliac disease. Digestion. 1994;55:243-6.

26. Smecuol E, Mauriño E, Vazquez H, Pedreira S, Niveloni S, Mazure R, et al. Gynaecological and obstetric disorders in coeliac disease: frequent clinical onset during pregnancy or the puerperium. Eur J Gastroenterol Hepatol. 1996;8:63-89.

27. Stang A: Critical evaluation of the Newcastle-Ottawa scale for the assessment of the quality of nonrandomized studies in meta-analyses. Eur J Epidemiol 2010;25:603-5.

28. Stroup DF, Berlin JA, Morton SC, Olkin I, Williamson GD, Rennie D, et al. Meta-analysis of observational studies in epidemiology: a proposal for reporting. JAMA. 2000;283:2008-12.

29. Tata LJ, Card TR, Logan RFA, Hubbard RB, Smith CJP, West J. Fertility and pregnancy-related events in women with celiac disease: a population-based cohort study. Gastroenterology. 2005;128:849-55.

30. Tiboni GM, de Vita MG, Faricelli R, Giampietro F, Liberati M. Serological testing for celiac diseasein women undergoingassisted reproduction techniques. Hum Reprod. 2006;21:376-9.

31. Zugna D, Richiardi L, Akre O, Stephansson O, Ludvigsson JF. A nationwide population-based to determine whether coeliac disease is associated with infertility. Gut. 2010;59:1471-5

Received 8/1/2014 Accepted 3/2/2014 